\title{
Dinâmica da acumulação de fitomassa e nutrientes na serapilheira sob plantios clonais de eucalipto
}

\section{Giovanno Radel de VARGAS ${ }^{1}$, Jonas Eduardo BIANCHIN², Hilbert BLUM², Wilson WAGNER ${ }^{2}$}

\author{
${ }^{1}$ Universidade Estadual do Centro-Oeste, Guarapuava, Paraná, Brasil \\ ${ }^{2}$ Universidade Federal do Paraná, Curitiba, Paraná, Brasil. \\ *E-mail:dinidio@gmail.com
}

Recebido em maio/2018; Aceito em outubro/2018.

\begin{abstract}
RESUMO: O objetivo geral deste trabalho foi estimar a quantidade de fitomassa e nutrientes estocada na serapilheira acumulada e taxas de decomposição da serapilheira acumulada ao longo do ano em plantios com diferentes materiais genéticos de eucalipto, em áreas submetidas ou não ao desbaste florestal. $\mathrm{O}$ trabalho foi conduzido em Itatinga-SP. O experimento foi um delineamento de parcelas subdivididas. As parcelas foram compostas por 8 clones e um plantio semental, sendo subparcelas áreas em que foi realizado o desbaste e áreas sem desbaste. Para estimativa da serapilheira acumulada foram realizadas coletas em cada estação do ano, entre junho de 2015 e março de 2016. Após as avaliações concluiu-se que existiu um padrão de acúmulo de serapilheira associado às estações, com maiores valores na primavera e verão. A fração folhas foi a mais representativa quanto à quantidade de serapilheira acumulada no solo. Os valores de acúmulo total de serapilheira variaram pouco entre os materiais genéticos avaliados. A decomposição foi ligeiramente maior nas áreas desbastadas comparativamente às áreas sem desbaste. A sequência decrescente dos teores nas frações foi: Folhas $>$ Galhos $>$ Casca $>$ Órgãos reprodutivos $>$ Outros materiais. Quanto aos elementos a sequência foi: $\mathrm{N}>\mathrm{Ca}>\mathrm{Mg}>\mathrm{K}>\mathrm{P}$. Palavras-chave: biogeoquímica florestal, serapilheira acumulada, desbaste, decomposição.
\end{abstract}

\section{Dynamics of litter stock and nutrients under clonal eucalyptus plantations}

\begin{abstract}
The objectives of this work were: to estimate the amount of phytomass and nutrients stored in the accumulated litter; to evaluate the seasonal variability of the amount of phytomass and nutrients in the accumulated litter and to estimate the decomposition rates of accumulated litter throughout the year. The work was conducted in Itatinga-SP. The experiment was an outline of subdivided plots. The plots were composed of 8 clones and one stallion planting, being subplots areas where thinning and non - roughing areas were performed. In order to estimate the accumulated litter, collections were made in each season of the year, between June 2015 and June 2016. After the evaluations it was concluded that there was a pattern of litter accumulation associated with the seasons, with higher values in spring and summer. The most representative leaf fraction was the amount of litter accumulated on the soil. Values of total litter accumulation were close between genetic materials. The decomposition was slightly higher in the thinned areas compared to the thinned areas. The decreasing sequence of the contents in the fractions was: Leaves $>$ Branches $>$ Bark $>$ Other materials $>$ Vegetable remains. As for the elements the sequence was: $\mathrm{N}>\mathrm{Ca}>\mathrm{Mg}>\mathrm{K}>\mathrm{P}$.
\end{abstract}

Keywords: forest biogeochemistry, litter stock, thinning, decomposition.

\section{INTRODUÇÃO}

A quantidade de fitomassa no solo compreende entre $20 \mathrm{e}$ $50 \%$ da matéria orgânica total na maioria dos ecossistemas florestais, aumentando ainda mais em alguns casos em florestas plantadas (SCHEER, 2008).

A serapilheira acumulada sobre o solo inclui todos os segmentos da planta depositados na superfície do solo, como folhas, caules, ramos, frutos, flores e os restos vegetais (VIERA; SCHUMACHER, 2010). Este material corresponde a uma reserva de matéria orgânica e nutrientes que influenciam diversos processos de funcionamento de um ecossistema florestal (POWERS et al., 2009).

Em determinadas situações este material presente na superfície do solo vêm a se tornar, após a sua decomposição, a principal fonte de nutrientes para o solo e para as plantas, via processo de ciclagem, garantindo oferta de nutrientes aos ecossistemas florestais (ZHANG et al., 2008).
A quantidade de nutrientes presentes na serapilheira acumulada e a sua disponibilização dependem das taxas de deposição e de decomposição do material oriundo da vegetação. Estes processos dependem de certos fatores como espécies e população presentes no ecossistema, atividade biológica, disponibilidade de água e umidade, eficiência dos processos biológicos, além da própria origem do elemento químico a ser disponibilizado após a decomposição (CHAGAS et al., 2012).

Em florestas plantadas, a prática do desbaste pode eventualmente influenciar na quantidade de fitomassa depositada sobre o solo. Segundo Wollum; Schubert (1975), quando da realização do desbaste em plantios de algumas espécies florestais, ocorrem alterações significavas na decomposição e mineralização do material da serapilheira, principalmente devido às alterações ambientais causadas com a retirada de árvores do ambiente, como diminuição da 
competição por espaço, água e nutrientes entre as plantas, abertura do dossel e aumento da incidência dos raios solares, alterando inclusive o microclima do ambiente, alterando, desta forma, a maneira como ocorre o acúmulo, deposição e decomposição de serapilheira nos ecossistemas florestais.

Segundo Rodrigues et al. (2010), o acúmulo de serapilheira é maior durante o período de desenvolvimento e crescimento inicial de um povoamento florestal, de maneira que esta tende a estabilizar-se e ocorrer de maneira constante conforme aumenta a maturidade e desenvolvimento das plantas. A temperatura normalmente tem efeito significativo na taxa de decomposição, geralmente ocorrendo redução na velocidade de decomposição ao longo de um período intenso de queda de temperatura, ao contrário do que ocorre em regiões com menores oscilações de temperatura, onde as taxas de decomposição normalmente são menos variáveis ao longo destes períodos.

Devido a influência do ambiente na composição da serapilheira, observa-se que espécies que crescem em solos mais férteis, possuem concentrações maiores de nitrogênio e fósforo, por exemplo; consequentemente produzindo serapilheira com quantidade maior destes nutrientes em comparação às regiões com solos menos férteis; o que sugere que estas espécies que se desenvolvem em situações mais adversas apresentam compostos de defesa em seus tecidos vegetais, diminuindo as taxas de decomposição do material gerado por estas espécies (LACLAU et al., 2008). Em povoamentos florestais com diferentes materiais genéticos é de se esperar que tanto a acumulação quanto a decomposição sejam influenciadas pela quantidade e pela qualidade de serapilheira produzida.

Algumas pesquisas relacionadas ao acúmulo e decomposição de serapilheira nos ecossistemas florestais tem sido realizadas com o intuito de compreender melhor a ciclagem biogeoquímica de nutrientes nos ecossistemas naturais e em plantios florestais, conforme os trabalhos realizados em plantios de eucalipto por Kolm; Poggiani (2003), Kolm (2001), Costa et al. (2005), Silva et al. (2012), Schumacher et al. (2013); além de diversas pesquisas relacionadas à dinâmica da serapilheira nos mais diversos ecossistemas florestais nativos (PEREIRA et al. 2008; SCHMIDT et al. 2008; BLUM, 2014; MEDEIROS, 2015; WOICIECHOWSKI, 2015).

Apesar da importância do eucalipto no contexto florestal brasileiro, são poucas as pesquisas buscando compreender o funcionamento biogeoquímico nos povoamentos florestais. E são raríssimas as pesquisas comparando diferentes materiais genéticos e avaliando o efeito do manejo florestal na ciclagem de nutrientes.

O objetivo geral deste trabalho foi estimar a quantidade de fitomassa e nutrientes estocada na serapilheira acumulada e taxas de decomposição da serapilheira acumulada ao longo do ano em plantios com diferentes materiais genéticos de eucalipto, em áreas submetidas ou não ao desbaste florestal.

Neste contexto, os objetivos específicos deste trabalho, foram: estimar a quantidade de fitomassa e os teores de nutrientes na serapilheira acumulada sobre o solo; avaliar a variabilidade estacional da quantidade de fitomassa e dos teores de nutrientes na serapilheira acumulada; estimar as taxas de decomposição da serapilheira acumulada durante as estações do ano.

\section{MATERIAL E MÉTODOS}

Este projeto foi desenvolvido na Estação Experimental de Ciências Florestais de Itatinga (EECFI), pertencente à "Escola Superior de Agricultura "Luiz de Queiroz" da Universidade de São Paulo (ESALQ/USP)", a qual está situada na região centro-sul do interior do estado de São Paulo, coordenadas $23^{\circ} 10^{\prime} 37^{\prime}$ S e $48^{\circ} 40^{\prime} 22^{\prime \prime} \mathrm{W}$, no município de Itatinga. A área da EECFI é de aproximadamente 2119,6 ha. O relevo da área é em sua maioria suave ondulado, com altitude aproximada de $850 \mathrm{~m}$, tendo predominantemente solos classificados como Latossolos Vermelhos de textura arenosa.

O clima é definido como temperado úmido com Inverno seco (Cwa), segundo a classificação de Köppen. A temperatura média anual da área é próxima dos $20{ }^{\circ} \mathrm{C}$ com temperaturas mínimas durante o ano em torno de $5{ }^{\circ} \mathrm{C}$ e temperaturas máximas em torno de $30{ }^{\circ} \mathrm{C}$ (INMET, 2017; CLIMATEDATA, 2015). A precipitação média anual é de $1300 \mathrm{~mm}$ e a região faz parte da bacia hidrográfica do Rio Paranapanema (ESALQ, 2014). A vegetação original da região é classificada como Floresta Estacional Semidecidual, pertencente predominantemente ao bioma Mata Atlântica (PISANI, 2009).

As áreas escolhidas para a realização da pesquisa são parcelas plantadas no ano de 2009, compostas por oito plantios clonais e um plantio semental de eucalipto com e sem desbaste (Figuras 1 e 2). O desbaste foi feito durante os meses de outubro e novembro de 2013. Foram escolhidos oito clones para o experimento, sendo estes definidos como C219, I-042, 1277, H13, GG100, I224, URO CAM, I144 e um plantio semental chamado de E. grandis espalhados aleatoriamente na área de avaliação do experimento.

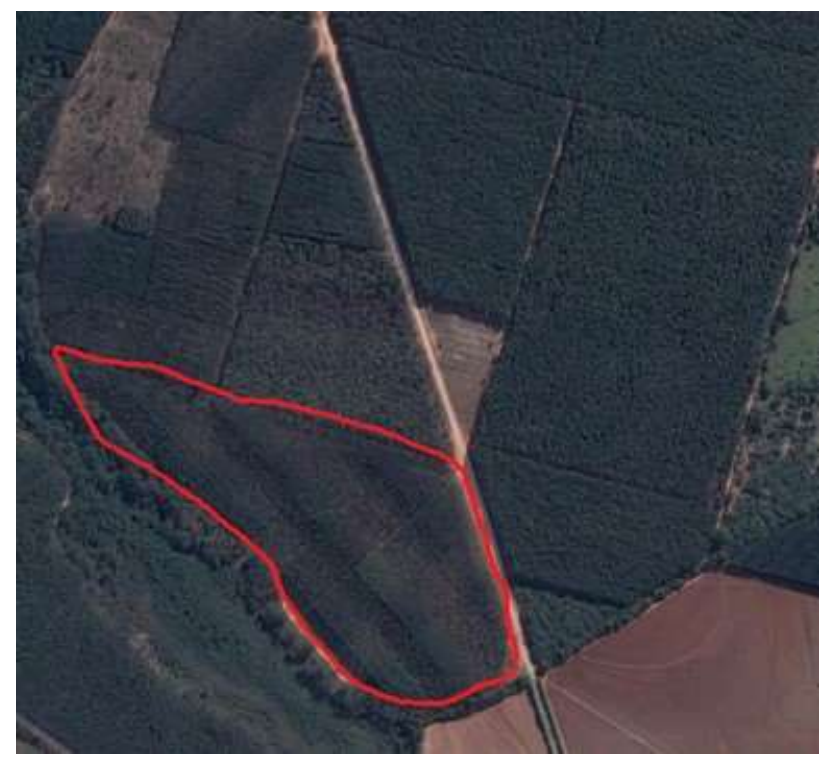

Figura 1. - Fotografia aérea da Estação Experimental de Ciências Florestais de Itatinga (Em destaque a área de estudo).

Figure 1. Aerial photograph of the Experimental Station of Forestry Sciences of Itatinga (In the area of study).

Para cada plantio clonal, foram consideradas 26 linhas com 20 árvores em cada linha, com espaçamento de 3,0 x 2,0 m entre si antes do desbaste e $26 \times 10$ plantas e espaçamento 3,0 x 4,0 m na área em que foi realizado desbaste. O plantio semental possui 20 linhas com 20 plantas em cada linha. Cada plantio clonal (parcela) é dividido em duas subparcelas de 13 linhas, onde nas 13 primeiras linhas de cada parcela não foi realizado o desbaste e nas 13 últimas linhas foi realizado o 
desbaste, o plantio semental é dividido da mesma maneira, mas com subparcelas de 10 linhas cada.

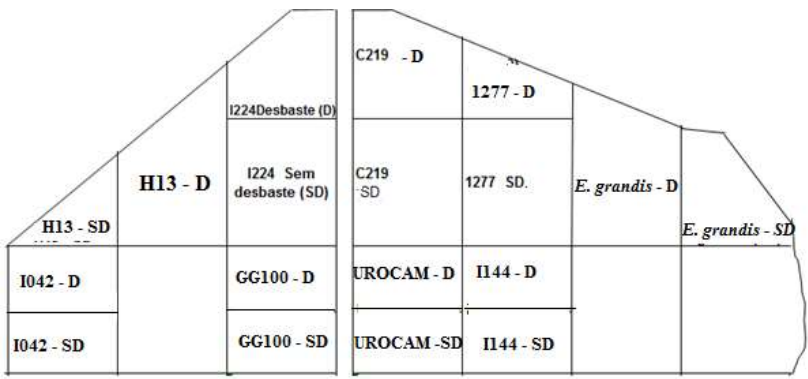

Figura 2. - Croqui do posicionamento dos plantios no campo. Figure 2. Sketch of the positioning of the plantations in the field.

O delineamento experimental foi em esquema fatorial 9x2 sendo 9 clones de eucalipto (cite eles aqui) e dois sistemas de condução (com e sem desbaste). As parcelas foram subdividas no tempo com coletas realizadas na primavera, verão, outono e inverno. No caso da serapilheira acumulada foram considerados 2 áreas de coleta por subparcela num total de 4 coletas por ano. Para comparações quanto ao efeito das estações do ano sobre a fitomassa e nutrientes da serapilheira acumulada, o número de repetições de cada tratamento foi equivalente ao número de coletores.

Duas áreas de 2,0 x 1,0 m (Figura 3) foram marcadas sobre o solo de cada subparcela (área com e sem desbaste) para a coleta de serapilheira acumulada, totalizando quatro áreas de coleta por parcela. As áreas de coleta foram posicionadas entre as linhas "5 e 6" e entre as árvores " 8 e 9" e "13 e 14" de cada subparcela, isoladas com fita de identificação. Antes do início das coletas, cada área de coleta teve todo o material de serapilheira removido, ficando o solo exposto. As coletas foram realizadas ao fim de cada estação climática (primavera, verão, outono e inverno) durante o período de junho de 2015 a março de 2016. A primeira coleta foi realizada 3 meses após a preparação da área para coleta e assim sucessivamente para as demais estações. A área de 2,0 x 1,0 m foi dividida em quatro quadrantes $(0,5 \times 1,0 \mathrm{~m})$ sendo a primeira coleta feita em um dos quadrantes, a segunda em outro dos 4 quadrantes; e, assim, sucessivamente, até a quarta coleta, sendo cada uma das coletas feitas em quadrantes distintos.

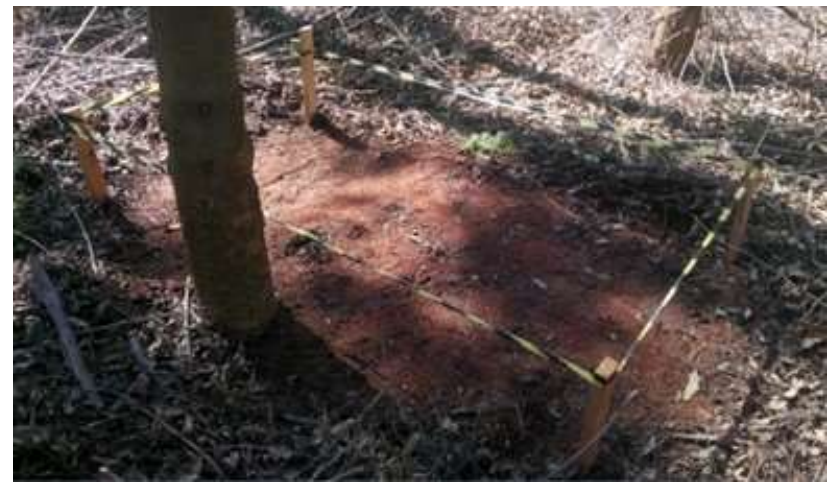

Figura 3. Área de coleta de serapilheira acumulada Figure 3. Collection area of accumulated litter.

A serapilheira coletada foi separada em frações (folhas, galhos, cascas, órgãos reprodutivos e outros materiais), para posterior secagem em estufa entre $60-70{ }^{\circ} \mathrm{C}$ até peso constante, sendo em seguida pesada para obtenção de sua massa seca e, só então, moída em moinho de facas (tipo Wiley) para realização das determinações químicas.

A estimativa da taxa de decomposição $(\mathrm{k})$ da serapilheira foi calculada a partir dos valores de produção anual de serapilheira (L) e de acúmulo sobre o solo (Xss) seguindo a Equação 1. Na sequência foi calculado o tempo médio de renovação da serapilheira, obtido através da estimativa $1 / \mathrm{k}$, sendo feitos os respectivos cálculos para cada clone e para o plantio semental (OLSON, 1963):

$$
k=\frac{L}{X s S}
$$

(Equação 1)

em que: $k=$ taxa de decomposição; $L=$ acúmulo sobre o solo produção anual de serapilheira; Xss = acúmulo de serapilheira sobre o solo

As amostras de serapilheira acumulada, uma vez na forma de pó, foram submetidas às determinações de fósforo $(\mathrm{P})$, potássio $(\mathrm{K})$, cálcio $(\mathrm{Ca})$ e magnésio $(\mathrm{Mg})$ (MARTINS; REISSMANN, 2007). Para determinação destes elementos químicos, o material vegetal foi incinerado em mufla a $500^{\circ} \mathrm{C}$ e posteriormente solubilizado em $\mathrm{HCl} 3 \mathrm{~mol} \mathrm{~L}^{-1}$.

O P foi determinado por colorimetria com vanadatomolibdato de amônio e leitura em espectrofotômetro Shimadzu, modelo UV/Vis 1240 Mini. As determinações de K foram realizadas por fotometria de chama.

Os elementos $\mathrm{Ca}$ e $\mathrm{Mg}$ foram determinados por espectrofotometria de absorção atômica. Para a obtenção dos teores de $\mathrm{C}$ e $\mathrm{N}$ foram pesados entre 15 e $20 \mathrm{mg}$ do material moído, em moinho de bola, sendo a determinação realizada por combustão em analisador CNHOS, marca ELEMENTAR $\AA$, modelo Vario EL III.

Os resultados foram submetidos à análise de variância e teste de Tukey ( $5 \%$ de significância), para comparação das médias (PIMENTEL-GOMES, 2009). As análises estatísticas foram realizadas com o emprego do software ASSISTAT, versão 7,7 (SILVA; AZEVEDO, 2016).

\section{RESULTADOS}

\subsection{Serapilheira Acumulada}

O acúmulo de serapilheira (Figuras 4 e 5), apresentou variação ao longo das estações climáticas, sendo primavera e verão as estações com maior acúmulo de material, apresentando, de maneira geral, valores bem próximos entre clones e entre áreas com e sem desbaste. As estações do outono e inverno apresentaram menor acúmulo, tanto na área com desbaste como na área sem desbaste. Os resultados são semelhantes aos observados por Vieira et al. (2014), em experimento comparando a deposição e acúmulo de serapilheira em alguns plantios clonais de Eucalipto.

Para as diferentes frações da serapilheira, poucas diferenças foram identificadas entre áreas com e sem desbaste (Tabela 1). E para a serapilheira total observa-se uma pequena tendência de valores mais elevados nas áreas em que não foi realizado o desbaste, o que era esperado pelo maior número de árvores nestas áreas. Estes resultados concordam com resultados obtidos por Vesterdal et al. (1995) em plantios de espécies pináceas na Noruega, e também por Kolm (2001), em plantios de eucaliptos sob desbastes progressivos no interior de São Paulo.

Houveram poucas diferenças para o acúmulo de serapilheira entre os diferentes materiais genéticos avaliados (Tabela 2), as principais diferenças observadas são 
comentadas na sequência. Para órgãos reprodutivos e casca o clone UROCAM acumulou menor quantidade de material. Para a fração galhos somente os clones 1277 e UROCAM mostraram valores mais baixos de acumulação. Já para a fração outros materiais E. grandis, C219 e UROCAM apresentaram valores inferiores aos demais. Quanto aos valores totais de acúmulo de serapilheira o clone UROCAM se destacou com menor acumulação de material, sendo este resultado influenciado pelo menor acúmulo das diversas frações de sua serapilheira.

Com relação à repartição de fitomassa entre as diferentes frações de serapilheira, esta segue de maneira geral, em relação aos valores brutos observados a seguinte sequência: Folhas $>$ Galhos > Orgãos Reprodutivos > Casca > outros materiais; para a maioria dos materiais genéticos avaliados.

3.2. Estimativas de decomposição da serapilheira

As taxas de decomposição variaram entre 0,98 e 1,27 (Tabela 3).

\subsection{Concentração de nutrientes na serapilheira acumulada}

Os teores médios anuais dos nutrientes na serapilheira acumulada, independentemente do material genético ou do tipo de fração da serapilheira, apresentaram a seguinte tendência de valores, sem terem sido observadas diferenças estatísticas: $\mathrm{N}>\mathrm{Ca}>\mathrm{Mg}>\mathrm{K}>\mathrm{P}$ (Tabela 4). Hernández et al. (2009), em experimento com Eucalyptus dunni no Uruguai, obtiveram sequência diferente nas folhas de alguns materiais clonais de eucalipto $(\mathrm{Ca}>\mathrm{K}>\mathrm{N}>\mathrm{Mg}>\mathrm{P})$, o que talvez possa ser explicado pela espécie diferente de eucalipto ou pelas diferentes características do solo onde foi feito o estudo no Uruguai.

Os teores $\mathrm{N}$ mostraram-se mais elevados nas folhas, entre 13,4 (I144) e 21,5 g kg-1 (C219); e nas demais frações da serapilheira (Galhos, Casca, Orgãos Reprodutivos e Restos) variaram entre 7,9 e $17,8 \mathrm{~g} \mathrm{~kg}^{-1}$.

Os teores de $\mathrm{P}$ variaram menos entre as diferentes frações; entre 0,6 e 1,0 $\mathrm{g} \mathrm{kg}^{-1}$, com os valores mais elevados sendo também observados nas folhas. $\mathrm{O} \mathrm{P}$ apresentou os menores teores entre os macronutrientes, assim como observado por Zaia; Gama-Rodrigues (2004) em estudo de ciclagem de nutrientes em alguns materiais clonais da mesma espécie. Segundo Bianchin et al, (2016), a maior parte da demanda de $\mathrm{P}$ nas árvores é suprida pelo ciclo biogeoquímico das mesmas, por meio de processos de remobilização do $\mathrm{P}$ que ocorre anteriormente à queda das folhas, o que explicaria em parte os valores mais baixos de $\mathrm{P}$ em relação aos demais nutrientes.

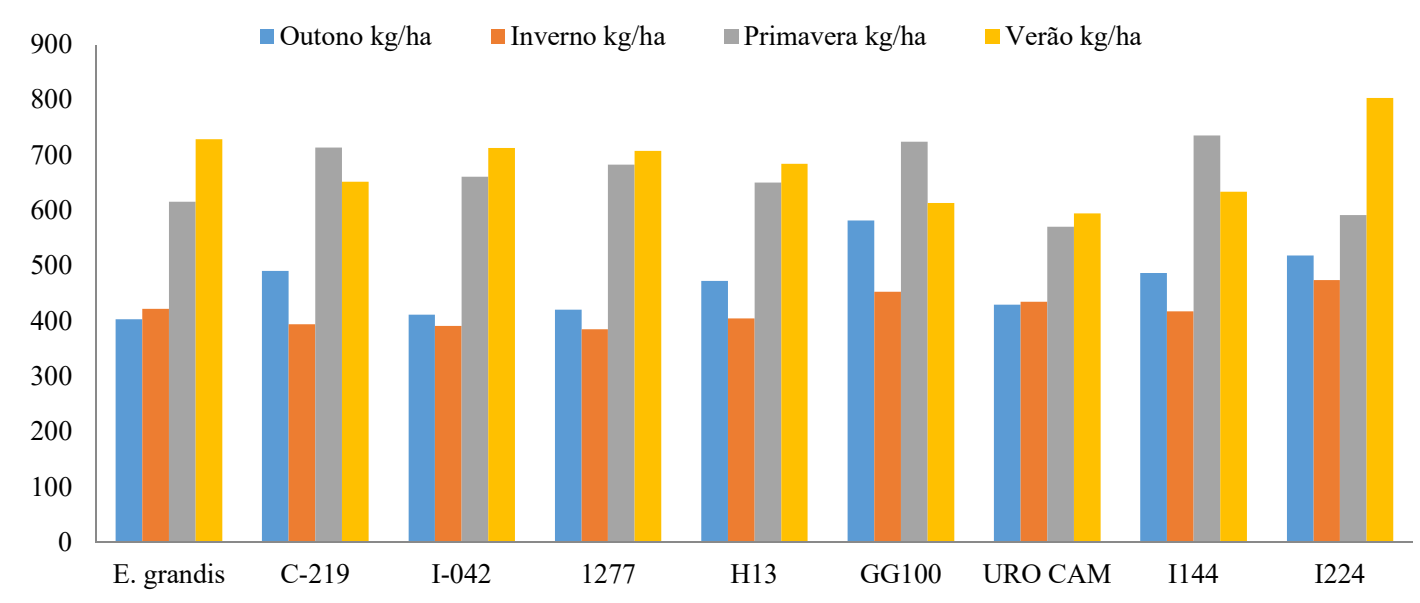

Figura 4. Acúmulo de serapilheira na área sem desbaste, por estação, entre junho de 2015 e março de 2016.

Figure 4. Accumulation of litter in the area without thinning, by season, between June 2015 and March 2016.

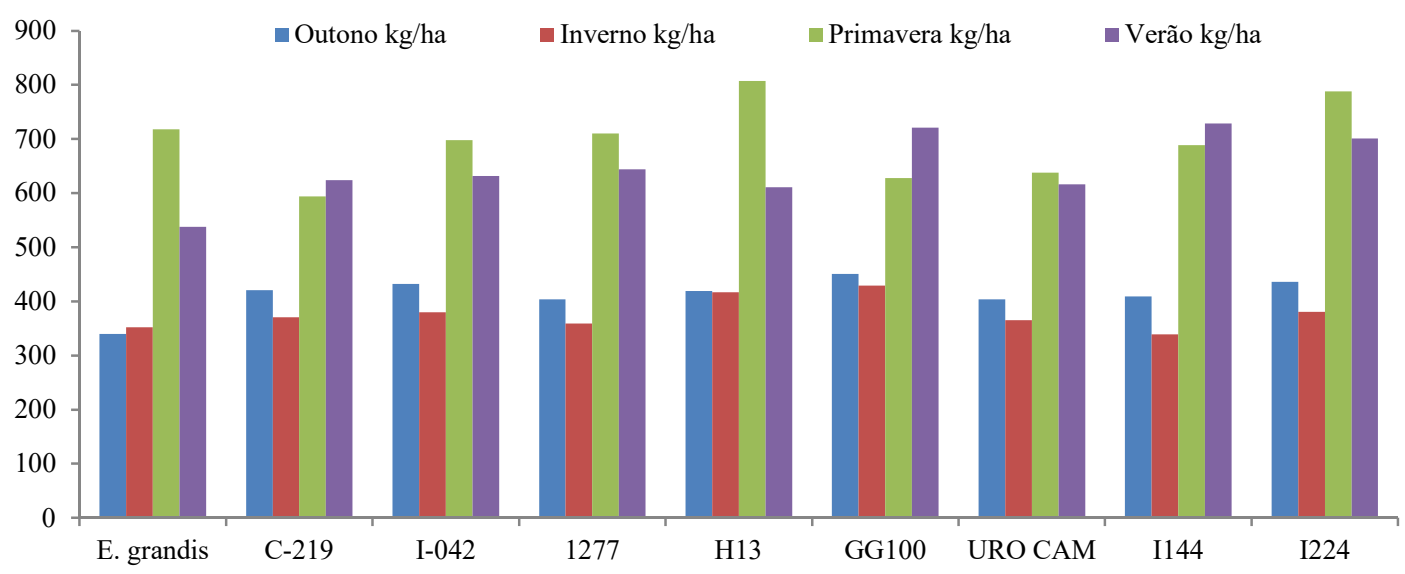

Figura 5. Acúmulo de serapilheira na área com desbaste, por estação, entre junho de 2015 e março de 2016. Figure 5. Accumulation of litter in the thinning area, by season, between June 2015 and March 2016. 
Tabela 1. Acumulo anual de serapilheira em área cultivada com diferentes clones de eucalipto e conduzidos em sistema de cultivo desbastado (D) e sem desbaste (SD) na região de Itatinga-SP.

Table 1. Annual accumulation of litter in an area cultivated with different eucalyptus clones and conducted in a finely chopped (D) and thinning (SD) system in the region of Itatinga-SP.

\begin{tabular}{|c|c|c|c|c|c|c|}
\hline \multirow{2}{*}{ Clones } & \multirow{2}{*}{ Desbaste } & \multicolumn{5}{|c|}{$\begin{array}{l}\text { Fitomassa }\left(\mathrm{kg} \mathrm{ha}^{-1}\right) \\
\end{array}$} \\
\hline & & Folhas & Órgãos Reprodutivos & Casca & Galhos & Outros Materiais \\
\hline \multirow[t]{2}{*}{ E. grandis } & Desbastado & 1592 & $241 \mathrm{~B}$ & 228 & $293 \mathrm{~B}$ & $127 \mathrm{~B}$ \\
\hline & Sem desbaste & 1426 & $522 \mathrm{~A}$ & 188 & $405 \mathrm{~A}$ & $222 \mathrm{~A}$ \\
\hline \multirow[t]{2}{*}{$\mathrm{C} 219$} & Desbastado & 1567 & 309 & 252 & $279 \mathrm{~B}$ & 152 \\
\hline & Sem desbaste & 1552 & 404 & 272 & $421 \mathrm{~A}$ & 216 \\
\hline \multirow[t]{2}{*}{$\mathrm{I}-042$} & Desbastado & 1565 & 369 & 234 & 345 & 214 \\
\hline & Sem desbaste & 1644 & 272 & 242 & 304 & 300 \\
\hline \multirow[t]{2}{*}{1277} & Desbastado & 1682 & 285 & 222 & 260 & 247 \\
\hline & Sem desbaste & 1681 & 304 & 228 & 270 & 314 \\
\hline \multirow[t]{2}{*}{ H13 } & Desbastado & 1599 & 298 & 230 & 354 & 337 \\
\hline & Sem desbaste & 1676 & 285 & 222 & 381 & 307 \\
\hline \multirow[t]{2}{*}{ GG100 } & Desbastado & 1682 & 272 & 233 & 354 & 297 \\
\hline & Sem desbaste & 1718 & 317 & 253 & 383 & 351 \\
\hline \multirow[t]{2}{*}{ UROCAM } & Desbastado & 1678 & 256 & 224 & 261 & $167 \mathrm{~B}$ \\
\hline & Sem desbaste & 1552 & 298 & 164 & 313 & $248 \mathrm{~A}$ \\
\hline \multirow[t]{2}{*}{$\mathrm{I} 144$} & Desbastado & 1673 & $264 \mathrm{~B}$ & 202 & 387 & 232 \\
\hline & Sem desbaste & 1698 & $400 \mathrm{~A}$ & 238 & 313 & 247 \\
\hline \multirow[t]{2}{*}{$\mathrm{I} 224$} & Desbastado & 1705 & 390 & 255 & 371 & 216 \\
\hline & Sem desbaste & 1721 & 332 & 317 & 416 & 255 \\
\hline
\end{tabular}

Médias seguidas de mesma letra na coluna não diferem estatisticamente pelo teste de Tukey à $5 \%$ de probabilidade. Letras maiúsculas comparam área com e sem desbaste em cada clone.

Tabela 2. Acúmulo anual médio de serapilheira total e nas diferentes frações em cada material genético.

Table 2. Average annual accumulation of total litter and the different fractions in each genetic material.

\begin{tabular}{ccccccc}
\hline \multirow{2}{*}{ Clones } & \multicolumn{3}{c}{ Fitomassa $\left(\mathrm{kg} \mathrm{ha}^{-1}\right)$} \\
\cline { 2 - 7 } & Folhas & Órgãos Reprodutivos & Casca & Galhos & OM & Total \\
\hline E. grandis & 1509 & $381 \mathrm{a}$ & $208 \mathrm{~b}$ & $349 \mathrm{a}$ & $174 \mathrm{~b}$ & $2622 \mathrm{a}$ \\
C219 & 1560 & $357 \mathrm{a}$ & $262 \mathrm{a}$ & $350 \mathrm{a}$ & $184 \mathrm{~b}$ & $2713 \mathrm{a}$ \\
I-042 & 1604 & $321 \mathrm{a}$ & $238 \mathrm{a}$ & $325 \mathrm{a}$ & $257 \mathrm{a}$ & $2745 \mathrm{a}$ \\
1277 & 1681 & $295 \mathrm{a}$ & $225 \mathrm{a}$ & $265 \mathrm{~b}$ & $281 \mathrm{a}$ & $2746 \mathrm{a}$ \\
H13 & 1637 & $292 \mathrm{a}$ & $226 \mathrm{a}$ & $367 \mathrm{a}$ & $322 \mathrm{a}$ & $2844 \mathrm{a}$ \\
GG100 & 1700 & $295 \mathrm{a}$ & $243 \mathrm{a}$ & $369 \mathrm{a}$ & $324 \mathrm{a}$ & $2930 \mathrm{a}$ \\
UROCAM & 1615 & $277 \mathrm{~b}$ & $194 \mathrm{~b}$ & $287 \mathrm{~b}$ & $208 \mathrm{~b}$ & $2581 \mathrm{~b}$ \\
I144 & 1686 & $332 \mathrm{a}$ & $220 \mathrm{a}$ & $350 \mathrm{a}$ & $239 \mathrm{a}$ & $2827 \mathrm{a}$ \\
I224 & 1713 & $361 \mathrm{a}$ & $286 \mathrm{a}$ & $393 \mathrm{a}$ & $236 \mathrm{a}$ & $2989 \mathrm{a}$
\end{tabular}

Médias seguidas de mesma letra na coluna não diferem estatisticamente pelo teste de Tukey à $5 \%$ de probabilidade. Quando há diferenças letras minúsculas comparam médias entre os clones em cada fração.

Tabela 3. Serapilheira depositada, acumulada, taxa de decomposição e tempo de renovação da serapilheira.

Table 3. Litter deposited, accumulated, rate of decomposition and time of renewal of litter.

\begin{tabular}{|c|c|c|c|c|c|}
\hline \multirow{3}{*}{ Clones } & \multirow{3}{*}{ Desbaste } & & & \multirow{3}{*}{$\mathrm{TD}(\mathrm{k})$} & \multirow{2}{*}{$\operatorname{TR}(1 / \mathrm{k})$} \\
\hline & & Depositada & Acumulada & & \\
\hline & & \multicolumn{2}{|c|}{$\mathrm{kg} \mathrm{ha} \mathrm{h}^{-1}$} & & Anos \\
\hline \multirow[t]{2}{*}{ E. grandis } & Desbastado & 2811 & 2480 & 1,13 & 0,88 \\
\hline & Sem desbaste & 3071 & 2762 & 1,11 & 0,90 \\
\hline \multirow[t]{2}{*}{ C219 } & Desbastado & 2899 & 2559 & 1,13 & 0,88 \\
\hline & Sem desbaste & 3206 & 2866 & 1,12 & 0,89 \\
\hline \multirow[t]{2}{*}{$\mathrm{I}-042$} & Desbastado & 2979 & 2727 & 1,09 & 0,92 \\
\hline & Sem desbaste & 3332 & 2763 & 1,21 & 0,83 \\
\hline \multirow[t]{2}{*}{1277} & Desbastado & 3132 & 2695 & 1,16 & 0,86 \\
\hline & Sem desbaste & 3119 & 2797 & 1,12 & 0,90 \\
\hline \multirow[t]{2}{*}{ H13 } & Desbastado & 3124 & 2819 & 1,11 & 0,90 \\
\hline & Sem desbaste & 3198 & 2870 & 1,11 & 0,90 \\
\hline \multirow[t]{2}{*}{ GG100 } & Desbastado & 3350 & 2838 & 1,18 & 0,85 \\
\hline & Sem desbaste & 3294 & 3023 & 1,09 & 0,92 \\
\hline \multirow[t]{2}{*}{ UROCAM } & Desbastado & 2652 & 2586 & 1,03 & 0,97 \\
\hline & Sem desbaste & 3269 & 2576 & 1,27 & 0,79 \\
\hline \multirow[t]{2}{*}{ I144 } & Desbastado & 3139 & 2758 & 1,14 & 0,88 \\
\hline & Sem desbaste & 2840 & 2897 & 0,98 & 1,02 \\
\hline \multirow[t]{2}{*}{$\mathrm{I} 224$} & Desbastado & 3543 & 2936 & 1,21 & 0,83 \\
\hline & Sem desbaste & 3099 & 3041 & 1,02 & 0,98 \\
\hline
\end{tabular}


Vargas et al.

Tabela 4. Teores médios (anual) de nutrientes na serapilheira acumulada por fração.

Table 4. Average (annual) levels of nutrients in litter accumulated per fraction.

\begin{tabular}{|c|c|c|c|c|c|}
\hline \multirow{2}{*}{ Clones } & \multicolumn{5}{|c|}{$\mathrm{N}\left(\mathrm{g} \mathrm{kg}^{-1}\right)$} \\
\hline & Folhas & Galhos & Casca & Órgãos Reprodutivos & Restos \\
\hline E. grandis & 19,0 & 10,3 & 16,2 & 15,9 & 9,7 \\
\hline C219 & 21,5 & 8,9 & 10,0 & 8,5 & 9,3 \\
\hline $\mathrm{I}-042$ & 20,1 & 15,5 & 14,6 & 15,6 & 15,3 \\
\hline 1277 & 16,1 & 12,8 & 12,3 & 13,4 & 17,8 \\
\hline $\mathrm{H} 13$ & 14,6 & 13,5 & 15,3 & 16,7 & 11,6 \\
\hline GG100 & 15,6 & 7,9 & 8,5 & 8,6 & 8,9 \\
\hline UROCAM & 17,2 & 16,4 & 15,9 & 15,4 & 15,0 \\
\hline I144 & 13,4 & 11,0 & 12,2 & & 11,9 \\
\hline I 224 & 14,0 & 17,8 & 10,3 & & 15,9 \\
\hline Clones & & & & & \\
\hline Clones & Folhas & Galhos & Casca & dutivos & Restos \\
\hline E. grandis & 0,9 & 0,6 & 0,7 & & 0,7 \\
\hline C219 & 0,9 & 0,6 & 0,5 & & 0,7 \\
\hline $\mathrm{I}-042$ & 0,9 & 0,4 & 0,5 & & 0,5 \\
\hline 1277 & 0,9 & 0,5 & 0,7 & & 0,6 \\
\hline H13 & 0,8 & 0,7 & 0,7 & & 0,7 \\
\hline GG100 & 1,0 & 0,5 & 0,6 & & 0,6 \\
\hline UROCAM & 0,8 & 0,4 & 0,7 & & 0,9 \\
\hline I144 & 1,0 & 1,1 & 1,1 & & 1,0 \\
\hline I 224 & 1,0 & 0,5 & 0,7 & & 0,6 \\
\hline & & & & & \\
\hline Clones & Folhas & Galhos & Casca & dutivos & Restos \\
\hline E. grandis & 1,4 & 1,7 & 1,8 & & 1,6 \\
\hline C219 & 1,6 & 1,4 & 2,2 & & 1,8 \\
\hline $\mathrm{I}-042$ & 1,3 & 1,8 & 1,9 & & 2,0 \\
\hline 1277 & 1,4 & 1,7 & 1,5 & & 1,4 \\
\hline H13 & 1,3 & 1,5 & 1,6 & & 1,3 \\
\hline GG100 & 1,3 & 2,3 & 1,5 & & 2,0 \\
\hline UROCAM & 1,4 & 1,2 & 2,1 & & 2,2 \\
\hline I144 & 1,3 & 2,5 & 1,4 & & 1,2 \\
\hline $\mathrm{I} 224$ & 1,4 & 1,6 & 1,7 & & 1,9 \\
\hline Clones & & & & & \\
\hline Clones & Folhas & Galhos & & Órgãos Reprodutivos & Restos \\
\hline E. grandis & 7,7 & 10,9 & & 5,0 & 9,4 \\
\hline C219 & 7,7 & 12,3 & & 11,1 & 11,6 \\
\hline $\mathrm{I}-042$ & 6,7 & 5,6 & & 5,0 & 5,2 \\
\hline 1277 & 8,0 & 5,9 & & 6,6 & 5,8 \\
\hline H13 & 11,0 & 8,5 & & 6,0 & 7,9 \\
\hline GG100 & 9,0 & 5,9 & & 9,6 & 12,5 \\
\hline UROCAM & 8,8 & 12,5 & & 10,5 & 11,0 \\
\hline I144 & 9,3 & 11,9 & & 9,6 & 13,2 \\
\hline I 224 & 8,9 & 10,6 & & 9,9 & 5,0 \\
\hline & & & & & \\
\hline Clones & Folhas & Galhos & & Órgãos Reprodutivos & Restos \\
\hline E. grandis & 5,7 & 2,2 & & 3,7 & 4,9 \\
\hline C219 & 4,6 & 3,5 & & 4,6 & 3,3 \\
\hline $\mathrm{I}-042$ & 4,6 & 2,8 & & 4,0 & 3,5 \\
\hline 1277 & 4,8 & 2,9 & & 3,3 & 3,7 \\
\hline H13 & 4,9 & 3,5 & & 3,4 & 4,2 \\
\hline GG100 & 4,6 & 4,0 & & 3,6 & 2,6 \\
\hline UROCAM & 7,0 & 3,0 & & 2,8 & 3,6 \\
\hline I144 & 5,5 & 4,6 & & 4,2 & 3,6 \\
\hline I 224 & 5,1 & 4,1 & & 3,7 & 3,7 \\
\hline
\end{tabular}

O potássio mostrou seus menores teores nas folhas $(1,3 \mathrm{a}$ $\left.1,6 \mathrm{~g} \mathrm{~kg}^{-1}\right)$ e nas demais frações variou entre 1,2 e $2,4 \mathrm{~g} \mathrm{~kg}^{-1}$. Por ser um nutriente muito móvel nas plantas, esperava-se que o K apresentasse maior variação em sua concentração nas frações de serapilheira ao longo do ano nas diferentes frações
(GIESSELMANN et al., 2010). Os valores relativamente baixos nas folhas, com valores próximos aos observados em materiais mais lenhosos como as cascas sugere que boa parte deste elemento já havia saído das folhas quando de sua deposição. Isto pode ser comprovado pelos teores de $\mathrm{K}$ nas 
folhas vivas das plantas, os quais são praticamente o dobro em comparação com os teores nas folhas de serapilheira (SCHEER et al., 2009).

Os teores de Ca foram superiores nas cascas, entre 10,2 e $12,9 \mathrm{~g} \mathrm{~kg}^{-1}$; e nas outras frações variaram entre 5,0 e 13,2 $\mathrm{g} \mathrm{kg}^{-}$

1. Teores elevados de $\mathrm{Ca}$ em cascas de eucalipto também foram observados por Gonçalves et al. (2000). Já o magnésio teve seus maiores teores nas folhas entre 4,6 e 7,0 $\mathrm{g} \mathrm{kg}^{-1}$, com as demais frações da serapilheira variando entre 2,2 e 5,6 $\mathrm{g} \mathrm{kg}^{-1}$. Os maiores valores obtidos na fração folha estão próximos do que normalmente é observado para o Eucalipto, conforme observado e citado por Silva et al. (2012). Segundo Towsend et al, (2008), concentrações nutricionais na serapilheira dependem de alguns fatores como espécie, frações da serapilheira e maneira como ocorre a translocação dos nutrientes nas plantas.

$\mathrm{Na}$ Tabela 5, observa-se, praticamente para todos os nutrientes e materiais genéticos, que não houveram diferenças estatísticas nos teores dos nutrientes na comparação entre áreas com e sem desbaste. A única diferença observada ocorreu no clone UROCAM para o $\mathrm{Ca}$, com teor mais elevado na área com desbaste.

Tabela 5. Concentração de nutrientes nas folhas da serapilheira acumulada, em povoamentos de eucalipto com desbaste (D) e sem desbaste (SD).

Table 5. Concentration of nutrients in the leaves of the accumulated litter, in eucalyptus plantations with thinning (D) and without thinning (SD).

\begin{tabular}{|c|c|c|c|c|c|c|c|c|c|c|}
\hline \multirow{2}{*}{ Clone } & \multicolumn{2}{|c|}{$\mathrm{N}$} & \multicolumn{2}{|c|}{$\mathrm{P}$} & \multicolumn{2}{|c|}{$\mathrm{K}$} & \multicolumn{2}{|c|}{$\mathrm{Ca}$} & \multicolumn{2}{|c|}{$\mathrm{Mg}$} \\
\hline & D & SD & D & SD & D & SD & D & SD & $\mathrm{D}$ & SD \\
\hline E. grandis & 18,0 & 20,1 & 0,8 & 1,0 & 1,3 & 1,6 & 8,0 & 7,4 & 6,0 & 5,4 \\
\hline $\mathrm{C} 219$ & 22,0 & 21,1 & 0,9 & 1,0 & 1,6 & 1,7 & 8,1 & 7,4 & 5,2 & 4,1 \\
\hline I-042 & 20,2 & 20,1 & 0,8 & 1,1 & 1,3 & 1,4 & 7,1 & 6,3 & 5,2 & 4,1 \\
\hline 1277 & 17,2 & 15,1 & 1,1 & 0,8 & 1,5 & 1,4 & 8,4 & 7,6 & 5,3 & 4,3 \\
\hline H13 & 15,0 & 14,3 & 0,9 & 0,7 & 1,5 & 1,2 & 12,0 & 10,1 & 4,6 & 5,2 \\
\hline GG100 & 16,2 & 15,1 & 1,1 & 1,0 & 1,4 & 1,3 & 10,1 & 8,0 & 4,0 & 5,3 \\
\hline UROCAM & 14,5 & 20,0 & 0,7 & 1,0 & 1,5 & 1,4 & $11,2 \mathrm{~A}$ & $6,4 \mathrm{~B}$ & 8,0 & 6,1 \\
\hline I144 & 11,3 & 15,6 & 1,0 & 1,0 & 1,5 & 1,2 & 10,4 & 8,2 & 6,0 & 5,0 \\
\hline I224 & 14,1 & 13,9 & 1,1 & 1,0 & 1,5 & 1,4 & 9,3 & 8,5 & 6,1 & 4,2 \\
\hline
\end{tabular}

Médias seguidas de mesma letra na linha não diferem estatisticamente pelo teste de Tukey à $5 \%$ de probabilidade. Quando há diferenças letras maiúsculas comparam área com e sem desbaste em cada clone.

\section{DISCUSSÃO}

Em alguns casos, (GIESSELMANN et al., 2011; SCHUMACHER et al., 2013; KOLM; POGGIANI, 2003) observa-se que espécies como o eucalipto depositam mais serapilheira nos meses mais quentes e úmidos e acumulam uma quantidade menor no mesmo período, devido à maior decomposição do material, consequentemente apresentando um acúmulo maior nas estações mais frias, onde a taxa de decomposição diminui.

Os maiores valores de acúmulo de serapilheira observados em períodos mais quentes e úmidos, como primavera e verão, discordam do que foi observado por Cunha et al. (2005) em plantios de eucalipto em rebrota de 1,5 e 5 anos no norte do Rio de Janeiro, que observou maior acúmulo de material nos períodos de menor disponibilidade hídrica.

$\mathrm{O}$ aumento na queda de folhas nos períodos mais úmidos e quentes normalmente ocorre devido às variações e ao aumento da temperatura, e da ocorrência de vento e precipitação, além da senescência natural das folhas (MAQUERE et al., 2008). Barlow et al. (2007), avaliando um plantio de Eucalyptus urophylla aos quatro e cinco anos de idade, encontraram aumento no acúmulo de folhas de serapilheira em períodos de maior precipitação mensal.

Vieira; Schumacher (2010a,b), estudando um povoamento de acácia negra, sugerem que a umidade do ar influenciou positivamente tanto a deposição quanto o acúmulo de serapilheira ao longo do ano, o que normalmente ocorre em períodos como verão e primavera na região do experimento.

Os valores aqui observados estão dentro da faixa de variação de outros estudos em povoamentos de eucalipto e em florestas subtropicais (ALHAMD, 2004; GRAHAM et al., 2010, CUNHA et al., 1993 CUNHA-NETO et al., 2013). Em plantios de eucalipto, normalmente os valores de decomposição encontram-se próximos de 1,0 (GUO et al., 2006). Valor mais elevado (próximo de 1,7) foi observado por Kolm; Poggiani (2003), em plantios de eucalipto mais velhos sob desbastes progressivos.

Alterações nos valores de deposição e acúmulo ao longo das estações podem ocorrer devido a aspectos cíclicos dos povoamentos durante estes períodos, além da influência de fatores climáticos e biológicos, como identificado por Sanches et al. (2009) em floresta tropical de transição.

Apesar de existirem diferenças na composição química (nutrientes) da serapilheira entre os materiais genéticos estudados (Tabela 4), estas não parecem ter sido suficientes para influenciar as taxas de decomposição entre os diferentes materiais genéticos.

Uma vez depositada e acumulada sobre o solo, o material da serapilheira passa por processos em que ocorre sua degradação, lavagem, ataque de microrganismos e consequentemente decomposição pela redução das frações minerais e húmicas (SILVA et al., 2008; VALENTI et al, 2008). Este processo é quase que totalmente biológico pela ação dos microrganismos presentes no solo, originando a ciclagem de nutrientes e o retorno destes ao solo, sendo controlado principalmente por fatores bióticos e abióticos como os teores de nutrientes no material e a própria atividade microbiana (DICKOW et al., 2008).

As alterações ambientais e na densidade de plantas, causadas pelo desbaste, até o momento não surtiram o efeito expressivo nas decomposições de serapilheira, o que também foi observado por Paula et al. (2009), em avaliações com diferentes fragmentos florestais.

Segundo Silva (1999), alterações causadas pelo desbaste, com o passar do tempo, tendem a alterar alguns fatores como evapotranspiração e aumento da radiação solar, favorecendo 
também a atividade dos microrganismos decompositores, mas até o momento da coleta de dados deste trabalho este efeito não se manifestou.

\section{CONCLUSÕES}

Ocorre um padrão de acúmulo de serapilheira associado às estações do ano, com maiores valores na primavera e verão.

A tendência de valores apresentada é que a fração foliar é a mais representativa em termos de fitomassa e a sequência decrescente de teores de nutrientes nas frações de serapilheira acumulada segue a seguinte tendência de valores: Folhas $>$ Galhos $>$ Casca $>$ Órgãos reprodutivos $>$ Outros materiais.

Para os nutrientes analisados a sequência de valores obtida é: $\mathrm{N}>\mathrm{Ca}>\mathrm{Mg}>\mathrm{K}>\mathrm{P}$.

\section{REFERÊNCIAS}

ALHAMD, L.; ARAKAKI, S.; HAGIARA, A. Decomposition of leaf litter of four tree species in a subtropical evergreen broad-leaved forest, Okinawa Island, Japan. Forest Ecology and Management, Amsterdam v. 202, v. n. 1-3, $\begin{array}{llll}\text { p. } & 1-11 & 2004 . & \end{array}$ https://dx.doi.org/10.1016/j.foreco.2004.02.062

BARLOW, J.; GARDNER, T. A.; FERREIRA, L. V.; PERES, C. A. Litter fall and decomposition in primary, secondary and plantation forests in the Brazilian Amazon. Forest Ecology and Management, Amsterdam, v. 247, n. 1-3, p. 91-97, 2007.

DOI: https://dx.doi.org/10.1016/j.foreco.2007.04.017

BIANCHIN, J. E.; MARQUES R.; BRITEZ R. M.; CAPRETZ, R. L. Deposição de fitomassa em formações secundárias na Floresta Atlântica do Paraná. Floresta e Ambiente, Seropédica, v. 23, n. 4, p. 524-533, 2016. DOI: http://dx.doi.org/10.1590/2179-8087.134015

BLUM, H. Caracterização biogeoquímica da serapilheira e de substâncias húmicas do solo sob plantios com espécies florestais nativas e exóticas no sul do Brasil. 2014. 113f. Tese (Doutorado em Engenharia Florestal) Universidade Federal do Paraná, Curitiba, 2014.

COSTA, G. S.; GAMA-RODRIGUES, A. C.; CUNHA, G. M. Decomposição e liberação de nutrientes da serapilheira foliar em povoamentos de Eucalyptus grandis no norte Fluminense. Revista Árvore, Viçosa, v. 29, n. 4, p.563570, 2005. DOI: http://dx.doi.org/10.1590/S010067622005000400008

CUNHA, G. M.; GAMA-RODRIGUES, A. C.; COSTA, G. S. Ciclagem de nutrientes em Eucalyptus grandis W. Hill ex Maiden no norte fluminense. Revista Árvore, Viçosa, v. 29, n. 3, p. 353-363, 2005. DOI: http://dx.doi.org/10.1590/S0100-67622005000300002

CUNHA, G. C.; GRENDENE, L. A.; DURLO, M. A. D.; BRESSAN, D. A Dinâmica nutricional em floresta estacional decidual com ênfase aos minerais provenientes da deposição da serrapilheira. Ciência Florestal, Santa Maria, v. 3, n. 1, p. 35-64, 1993. DOI: http://dx.doi.org/10.5902/19805098284

CUNHA-NETO, F. V. C.; LELES, P. S. S.; PEREIRA, M. G.; BELlUMATH, V. G. H.; ALONSO, J. M. Acúmulo e decomposição da serapilheira em quatro formações florestais. Ciência Florestal, Santa Maria, v. 23, n. 3, p. 379-387, 2013.

DOI: http://dx.doi.org/10.5902/1980509810549

CHAGAS, G. F. B. das; SILVA, V. de P. R. da; COSTA, A C. L. da; DANTAS, V. de A. Impactos da redução da pluviometria na biomassa aérea da Floresta Amazônica. Revista Brasileira de Engenharia Agrícola e Ambiental, Campina Grande, v. 16, n. 1, p. 1266-1274, 2012. DOI: http://dx.doi.org/10.1590/S141543662012000100010

DICKOW, K. M. C.; MARQUES, R.; PINTO, C. B. Lixiviação de nutrientes da serapilheira recém-depositada em sucessão ecológica na Floresta Atlântica, litoral do Paraná. Floresta, Curitiba, v. 39, n. 1, p. 145-156. 2008. DOI: http://dx.doi.org/10.5380/rf.v39i1.13734

GIESSELMANN, U. C.; MARTINS, K. G.; BRÄNDLEA, M.; SCHÄDLERC, M.; MARQUES, R.; BRAND, R. Lack of home-field advantage in the decomposition of leaf litter in the Atlantic Rainforest of Brazil. Applied Soil Ecology, Amsterdam, v. 49, p. 5-10. 2011. DOI: https://dx.doi.org/10.1016/j.apsoil.2011.07.010

GIESSELMANN, U. C.; MARTINS, K. G.; BRANDLEA, M.; SCHADLEC, M.; MARQUES, R.; BRAND, R. Diversity and ecosystem functioning: Litter decomposition dynamics in the Atlantic Rainforest. Applied Soil Ecology, Amsterdam, v. 46, p. 283-290, 2010. DOI: https://dx.doi.org/10.1016/j.apsoil.2010.07.006

GONÇALVES, J. L. M.; STAPE, J. L.; BENEDETTI, V.; FESSEL, V. A. G.; GAVA, J. L. Reflexos do cultivo mínimo e intensivo do solo em sua fertilidade e na nutrição das árvores. In: GONÇALVES, J.L.M.; BENEDETTI, V., (Ed.) Nutrição e fertilização florestal. Piracicaba: IPEF, 2000. p. 1-57.

GRAHAM, E. A.; LAM, Y.; YUEN, E. M. Forest understory soil temperatures and heat flux calculated using a Fourier model and scaled using a digital camera. Agricultural and Forest Meteorology, Amsterdam, v. 150, n. 4, p. 640-649, 2010. DOI: https://dx.doi.org/10.1016/j.agrformet.2010.02.005

GUO, L. B.; SIMS, R. E. H.; HORNE, D. J. Biomass production and nutrient cycling in Eucalyptus short rotation energy forests in New Zealand: II., litter fall and nutrient return. Biomass and Bioenergy, Oxford, v. 30, n. 5, p. 393-404, 2006. https://doi.org/10.1016/j.biombioe.2005.11.017

HERNÁNDEZ, J.; PINO, A.; SALVO, L.; ARRARTE, G. Nutrient export and harvest residue decomposition patterns of a Eucalyptus dunnii Maiden plantation in temperate climate of Uruguay. Forest Ecology and Management, Amsterdam, v. 258, n. 2, p. 92-99, 2009. DOI: https://dx.doi.org/10.1016/j.foreco.2009.03.050

KOLM, L.; POGGIANI, F. Ciclagem de nutrientes em povoamentos de Eucalyptus grandis submetidos à prática de desbastes progressivos. Scientia Forestalis, Piracicaba, n. 63, p. 79-93, 2003.

KOLM, L. Ciclagem de nutrientes e variações do microclima em plantações de Eucalyptus grandis Hill ex Maiden manejadas através de desbastes progressivos. 2001. 88f. Dissertação (Mestrado em Ciências Florestais) - Universidade de São Paulo, São Paulo, 2001.

LACLAU, J. P.; JULIO C. R.; ALMEIDA, J. L.; GONCALVES, M.; SAINT-ANDRE, L.; VENTURA, M.; RANGER, J.; MOREIRA, R. Influence of nitrogen and potassium fertilization on leaf lifespan and allocation of above-ground growth in Eucalyptus plantations. Tree Physiology, Oxford, v. 29, n. 1, p. 111-124, 2008. DOI: https://dx.doi.org/10.1093/treephys/tpn010 
MAQUERE, V.; LACLAU, J. P.; BERNOUX, M.; SAINTANDRE, L.; GONÇALVES, J. L. M.; CERRI, C. C.; PICCOLO, M. C.; RANGER, J. Influence of land use (savanna, pasture, Eucalyptus plantations) on soil carbon and nitrogen stocks in Brazil. European Journal of Soil science, Oxford, v. 59, n. 5, p. 863-877, 2008. DOI: https://dx.doi.org/10.1111/j.1365-2389.2008.01059.x

MARTINS, A. P. L.; REISSMANN, C. B. Material vegetal e as rotinas laboratoriais nos procedimentos químicoanalíticos. Scientia Agraria, Curitiba, v. 8, n. 1, p. 1-17, 2007. DOI: http://dx.doi.org/10.5380/rsa.v8i1.8336

MEDEIROS, F. M. Ciclagem de nutrientes em estágios sucessionais da Floresta Ombrófila Densa do Paraná 2015, 144f. Tese (Doutorado em Engenharia Florestal) Universidade Federal do Paraná, Curitiba, 2015.

OLSON, J. S. Energy storage and the balance of producers and decomposers in ecological systems. Ecology, Washington, v. $44, \quad$ n. 2 , p. 322-331, 1963. DOI: https://dx.doi.org/10.2307/1932179

PAULA, R. R; PEREIRA, M. G.; MENEZES, L. F. T. Aporte de nutrientes e decomposição da serapilheira em três fragmentos florestais periodicamente inundados na Ilha de Marambaia, RJ. Ciência Florestal, Santa Maria, v. 19, n. 2, p. 139-148, 2009. DOI http://dx.doi.org/10.5902/19805098405

PEREIRA, M. G.; MENESES, L. F. T.; SCHULTZ, N. Aporte e decomposição da serapilheira na Mata Atlântica, ilha de Marambaia, Mangaratiba, RJ. Ciência Florestal, Santa Maria, v. 18, n. 4, p. 443-454, 2008. DOI: http://dx.doi.org/10.5902/19805098428

PIMENTEL-GOMES, F. Curso de estatística experimental. 15. Ed. Piracicaba: FEALQ, 2009. 451 p.

POWERS, J. S.; MONTGOMERY, R. A.; ADAIR, E. C.; BREARLEY, F. Q.; DEWALT, S. J.; CASTANHO, C. T. Decomposition in tropical forests: a pan-tropical study of the effects of litter type, litter placement and mesofaunal exclusion across a precipitation gradient. Journal of Ecology, Oxford, v. 97, n. 4, p. 801-11, 2009. DOI: https://dx.doi.org/10.1111/j.1365-2745.2009.01515.x

RODRIGUES, B. D.; MARTINS, S. V.; LEITE, H. G. Avaliação do potencial da transposição da serapilheira e do banco de sementes do solo para restauração florestal em área degradada. Revista Árvore, Viçosa, v. 34, n. 1, p. $65-$ 73, 2010. DOI: http://dx.doi.org/10.1590/S010067622010000100008

SANCHES, L.; VALENTINE, C. M. A.; BIUDES, M. S.; NOGUEIRA, J. S. Dinâmica sazonal da produção e decomposição de serapilheira em Floresta tropical de transição. Revista Brasileira de Engenharia Agrícola e Ambiental, Campina Grande, v. 13, n. 2, p. 183-189, 2009. DOI: http://dx.doi.org/10.1590/S141543662009000200012

SCHEER, M. B.; GATTI, G.; WISNIEWSKI, C.; MOCOCHINSKI, A. Y.; CAVASSANI, A. T.; LORENZETTO, A.; PUTINI, F. Patterns of litter production in a secondary alluvial Atlantic Rain Forest in southern Brazil. Revista Brasileira de Botânica, v. 32, n. 4, p. $\quad 805-817, \quad 2009$. DOI: http://dx.doi.org/10.1590/S0100-84042009000400018

SCHEER, M. B. Decomposição e liberação de nutrientes da serapilheira foliar em um trecho de floresta ombrófila densa aluvial em regeneração, Guaraqueçaba (PR).
Floresta, Curitiba, v. 38, n. 2, p. 253-266, 2008. DOI: http://dx.doi.org/10.5380/rf.v38i2.11620

SCHMIDT, P.; DICKOW, K. M. C.; ROCHA, A. A.; MARQUES, R.; SCHEUERMANN, L.; RÖMBKE, J.; FÖRSTER, B.; HÖFER, H. Soil macrofauna and decomposition rates in Southern Brazilian Atlantic Rainforests. Ecotropica, Cuiabá, v.14, p.89-100, 2008.

SCHUMACHER, M. V.; CORREA, R. S.; VIERA, M.; ARAUJO, E. F. Produção e decomposição de serapilheira em um povoamento de Eucalyptus urophylla $\times$ Eucalyptus globulus maidenii. Cerne, Lavras, v. 19, n. 3, p. 509-508, 2013. DOI: http://dx.doi.org/10.1590/S010477602013000300018

SILVA, F. de A. S.; AZEVEDO, C. A. V. de. The Assistat Software Version 7.7 and its use in the analysis of experimental data. African Journal of Agricultural Research, v. 11, n. 39, p. 3733-3740, 2016. DOI: https://dx.doi.org/10.5897/AJAR2016.11522

SILVA, J. V.; NOGUEIRA, G. S.; SANTANA, R. C.; LEITE, H. G.; OLIVEIRA, M. L. R.; ALMADO, R. P. Produção e acúmulo de nutrientes em povoamento de eucalipto em consequência da intensidade do desbaste e da fertilização. Pesquisa agropecuária brasileira, Brasília, v. 47, n. 11, p. 1555-1562, 2012.

SILVA, L. S.; RHODEN, A. C.; POCOJESKI, E.; CAMARGO, F. O. A.; BENEDETTI, E. Modelos matemáticos para a estimativa do potencial de mineralização anaeróbia do nitrogênio em solos de várzea do Rio Grande do Sul. Revista Brasileira de Ciência do Solo, Viçosa, v. 32, n. 4, p. 1513-1520, 2008

SILVA, L. F. Necessidade de adubação pós desbaste, baseada no balanço nutricional de povoamentos de eucalipto. Viçosa, 1999. 61f. Dissertação (Mestrado em Ciência do Solo) - Universidade Federal de Viçosa, Viçosa, 1999.

TOWNSEND, A. R.; ASNER, G. P.; CLEVELAND, C. C.; The biogeochemical heterogeneity of tropical forests. Ecology and Evolution, Amsterdam, v. 23, n. 8. p. 424434, 2008.2 DOI: https://dx.doi.org/10.1016/j.tree.2008.04.009

VALENTI, M. W.; CIANCIARUSO, M. V.; BATALHA, M. A. Seasonality of litterfall and leaf decomposition in a cerrado site. Brazilian Journal of Biology, São Carlos, v. 68, p. 459-465, 2008. http://dx.doi.org/10.1590/S1519-69842008000300002

VESTERDAL, L.; DALSGAARD, M.; FELBI, C.; RAULUND-RASMUSSEN, K.; JORGENSEN, B.B. Effects on thinning and soil properties on accumulation of carbon, nitrogen and phosphorus in the forest floor of Norway spruce stands. Forest Ecology and Management, v. 77, n. 1-3, p. 1-10, 1995. DOI: https://dx.doi.org/10.1016/0378-1127(95)03579-Y

VIEIRA, M.; SCHUMACHER, M. V.; ARAUJO, E. F.; CORREA, R. S.; WINCLER, M. V.; CALDEIRA, W. Deposição de Serapilheira e Nutrientes em Plantio de Eucalyptus urophylla $\times$ E. globulus. Floresta e Ambiente, Seropédica, v. 21, p. 327-338, 2014. DOI: http://dx.doi.org/10.1590/2179-8087.053913

VIEIRA, M.; SCHUMACHER, M. V. Deposição de serapilheira e de macronutrientes em um povoamento de acácia negra (Acacia mearnsii De Wild.) no Rio Grande do Sul. Ciência Florestal, v.20, p.225-233, 2010a. 
Vargas et al.

VIEIRA, M.; SCHUMACHER, M. V. Teores e aporte de nutrientes na serapilheira de pinus taeda 1., e sua relação com a temperatura do ar e pluviosidade Revista Árvore, v.34, p.85-94, 2010 b.

WOICIECHOWSKI, T. Ciclagem de fitomassa e nutrientes na Floresta Ombrófila Densa Submontana no litoram do Paraná. 2015. 113f. Tese (Doutorado em Engenharia Florestal) - Universidade Federal do Paraná, Curitiba, 2015.

WOLLUM, A. G.; SCHUBERT, G. H. Effect of thinning on the foliage and forest floor properties of ponderosa pine stands. Soil Science Society of America Journal, Madison, v. 39, n. 5, p. 968-972, 1975. DOI: https://dx.doi.org/10.2136/sssaj1975.0361599500390005 0044x
ZAIA, F. A.; GAMA-RODRIGUES, A. C. Ciclagem e balanço de nutrientes em povoamentos de eucalipto na região norte-fluminense. Revista Brasileira de Ciência do Solo, Viçosa, v. 28, n. 5, p. 843-852, 2004. DOI: http://dx.doi.org/10.1590/S0100-06832004000500007

ZHANG, D.; HUI, D.; LUO, Y.; ZHOU, G. L. Rates of litter decomposition in terrestrial ecosystems: global patterns and controlling factors. Journal of Plant Ecology, v. 1, n. 2, p. 85-93, 2008. DOI: https://dx.doi.org/10.1093/jpe/rtn002 